Highly Energetic Physical Processes and

Mechanisms for Emission from Astrophysical Plasmas

IAU Symposium, Vol. 195, 2000

P. C. H. Martens, S. Tsuruta, and M. A. Weber, eds.

\title{
Neutron Star Powered Accelerators
}

\section{Ruderman}

Dept. of Physics, Columbia University, New York, NY 10027, U.S.A.

\section{Magnetospheric Accelerators}

Neutron stars can be the underlying source of energetic particle acceleration in several ways. The huge gravitational-collapse energy released in their birth, or the violent fusion at the end of the life of a neutron-star binary, is the energy source for an accelerator in the surrounding medium far from the star. This would be the case for: (a) cosmic rays from supernova explosions with neutronstar remnants; (b) energetic radiation from "plerions" around young neutron stars (e.g., the Crab Nebula, see Pacini 2000); and (c) "afterglow" and $\gamma$-rays of cosmic Gamma-Ray Burst (GRB) sources with possible neutron-star central engines. Particles can also be energetically accelerated if a neutron star's gravitational pull sustains an accretion disk fed by a companion. Examples are accretion-powered X-ray pulsars and low-mass X-ray binaries. A third family of "neutron-star powered" accelerators consists of those which do not depend on the surrounding environment. These are the accelerators which must exist in the magnetospheres of many solitary, spinning-down, magnetized neutron stars ("spinsters") when they are observed as radio pulsars or $\gamma$-ray pulsars. (There are probably $\sim 10^{3}$ dead radio pulsars for each one in our Galaxy that is still active; the ratio for $\gamma$-ray pulsars may well exceed $10^{5}$.)

Despite over three decades of study, there is still no consensus on the geometry and structure of these magnetospheric accelerators and on that of the high-energy photon beams they emit. There is a general consensus, however, that these accelerators must be located on the bundle of "open" magnetic field lines coming out of the surface of the spinning star. These are the field lines which never return to the star; in a vacuum (not at all the case in a pulsar magnetosphere), these are the field lines which connect to the radiation field. More generally, for a star with angular spin $\boldsymbol{\Omega}$, the open field lines are those which pass through the "light cylinder" where $|\boldsymbol{\Omega} \times \mathbf{r}|=c$. "Closed" field lines return to the star before penetrating through the light cylinder. A corotating observer sees a static charge distribution only on the closed field lines; in the laboratory frame, this charge density corotates with the star. The near magnetosphere and its charge distribution act like a rigid extension of the star with one crucial difference. Inside the conducting star,

$$
\mathbf{E}-\left(\frac{\boldsymbol{\Omega} \times \mathbf{r}}{c}\right) \times \mathbf{B}=0 .
$$

There is then some net charge separation:

$$
\rho=\frac{\nabla \cdot \mathbf{E}}{4 \pi}=\frac{\boldsymbol{\Omega} \cdot \mathbf{B}}{2 \pi c}+\mathcal{O}(\nabla \times \mathbf{B}),
$$


a net separation less than $10^{-20}$ of that of the electrons inside the star. In the nearby, closed magnetosphere, where $\nabla \times \mathbf{B}$ is quite negligible,

$$
\rho=\frac{\boldsymbol{\Omega} \cdot \mathbf{B}}{2 \pi c} \equiv \rho_{\mathrm{GJ}}
$$

is the "Goldreich-Julian density". However, when the mass-dependent gravitational and centrifugal forces are included, the magnetospheric $\rho_{\mathrm{GJ}}$ becomes "charge-separated": where $\rho_{\mathrm{GJ}}<0$, only electrons are present, and where $\rho_{\mathrm{GJ}}>0$, there are only positive ions-all of which have the same charge-to-mass ratio. Where $\rho=\rho_{\mathrm{GJ}}, \mathbf{E} \cdot \hat{\mathbf{B}} \sim 0$, so there is no significant particle accelerator in the closed field-line part of the neutron-star magnetosphere, but, on the open field-line bundle, this is not the case. Steady current flow, $\mathbf{E} \cdot \mathbf{B} \sim 0$, and a charge-separated $\rho_{\mathrm{GJ}}$ are not compatible. Where $\rho=\rho_{\mathrm{GJ}}, \mathbf{E} \cdot \hat{\mathbf{B}} \neq 0$, and a particle accelerator along $\mathbf{B}$ forms. Most studies and models have focused on two critical locations along the open field-line bundle where such accelerators might be expected.

One such model is the polar-cap accelerator. Very near or at the stellar surface, a charge deficiency $|\rho|<\left|\rho_{\mathrm{GJ}}\right|$ can occur on a fraction of the open field-line bundle because of $B$ field-line curvature. In addition, where ions pulled from the stellar surface are the main, initial source for charged-particle current flow out along the open field-line bundle, the large ion inertia would give an excess of positive ions at the stellar surface. The resulting space-charge field could accelerate ions above that region to extreme relativistic energies. Surface matter structure in the huge $B>10^{14} \mathrm{G}$ field of a "magnetar" (see Thompson 2000) may cause $\rho<\left|\rho_{\mathrm{GJ}}\right|$ just above the stellar surface because of suppression of the need for outflow of positive ions. Inside all of these polar-cap (PC) accelerators, extreme relativistic $e^{-}\left(e^{+}\right)$moving along the curved field lines would be a source of "curvature $\gamma$-rays" with energies exceeding $10^{2} \mathrm{MeV}$ and perhaps even reaching to near $10 \mathrm{GeV}$. These $\gamma$-rays would make $e^{ \pm}$pairs when crossing local B-field lines $\left(\gamma+\mathbf{B} \rightarrow \mathbf{B}+e^{-}+e^{+}\right)$, or, depending on the intensity of local keV $\mathrm{X}$-ray (X) fluxes, by $\gamma+\mathrm{X} \rightarrow e^{-}+e^{+}$. The acceleration of $e^{+}$and $e^{-}$from such pairs can then lead to more $\gamma$-rays and $e^{ \pm}$pairs. If too many $e^{ \pm}$pairs are made within the accelerator, the accelerator is quenched enough to reduce that pair production. If not enough are produced to supply the charge lost to outward current flow, the accelerating region would grow larger and stronger until the $e^{ \pm}$production needed to accomplish it is reached.

The other focus of interest for open field-line accelerator locations is near the "null surface", where $\boldsymbol{\Omega} \cdot \mathbf{B}=0$ and $\rho_{\mathrm{GJ}}$ reverses sign. The null surface within the neutron star's open field-line bundle is a substantial fraction of the light cylinder's radius ( $c / \Omega \sim 10^{3}-10^{4}$ stellar radii) unless the star's dipole moment $\mu$ and spin $\boldsymbol{\Omega}$ are almost orthogonal.

For historic reasons, these outer-magnetosphere accelerators are often called "outer-gap" (OG) accelerators as distinguished from the PC ones. In OG accelerators, the needed $e^{ \pm}$production, which is the basic cause for their formation, is thought to be maintained by $\gamma+\mathrm{X} \rightarrow \mathrm{e}^{-}+\mathrm{e}^{+}$; the $\gamma$-rays, as in a $\mathrm{PC}$ accelerator, are curvature radiation from (oppositely accelerated) $e^{-}$and $e^{+}$, but now coming from pairs created within the OG accelerator far from the star. A crucial difference between PC and OG accelerators is in the maximum power 
each could give to maintain the expected maximum current density through the accelerator

$$
\mathbf{j}_{\mathrm{GJ}} \equiv \rho_{\mathrm{GJ}} c \hat{\mathbf{B}} \sim \frac{\Omega B}{2 \pi} \hat{\mathbf{B}} .
$$

To produce the $10^{2}-10^{3} \mathrm{MeV} \gamma$-rays to maintain this current, a PC accelerator usually needs a potential drop along $\mathbf{B}$ such that $\Delta V \sim$ a few times $10^{12}$ volts. If it is much greater, so many $e^{ \pm}$pairs would be produced that the $\mathrm{PC}$ accelerator would be quenched. For an OG accelerator to satisfy these same requirements, $\Delta V \sim 10^{14}$ volts. The extra factor of $10^{2}$ is from a combination of two circumstances. 1) Because only $\gamma+\mathrm{X} \rightarrow \mathrm{e}^{-}+\mathrm{e}^{+}$is effective in making pairs within an OG accelerator, a significant fraction of the $10^{5}$ curvature $\gamma$-rays from each $e^{-} / e^{+}$passing through most OG accelerators must have an energy of at least a GeV. 2) More importantly, inside OG accelerators, the energy of accelerated $e^{-} / e^{+}$is curvature-radiation reaction limited. The electrons do not ever acquire more than about $10^{-2}$ of the energy $e \Delta V$, so that an outergap accelerator needs $10^{2}$ times more $\Delta V$ than a $\mathrm{PC}$ one to achieve similar curvature-radiation properties.

For this menu of accelerator locations, the important choices to be made are:

a) Where are the accelerators responsible for radio-frequency emission from radio pulsars?

b) Where are the accelerators which power the $\gamma$-rays from $\gamma$-ray pulsars?

c) What is the radiation beam geometry (e.g., cones, fans, numbers, directions)?

d) How is the observed radiation emitted and, perhaps, processed before observation?

I think there is considerable observational support for believing that both PC and OG accelerators can exist in the same pulsar but that they fight each other for survival. Each may dominate on opposite sides of the star. Among the main arguments that PC accelerators power the coherent radio emission of canonical radio pulsars are the following. a) Radio-emission beam widths are usually quite narrow-just about what would be expected from extreme relativistic chargedparticle flow out along the open field-line bundle at radii very much less than the light-cylinder radius $c \Omega^{-1}$. (See, however, Lyutikov, Blandford, \& Machabeli 2000.) b) Radio emission from pulsars is observed to die out when these neutron stars have spun-down so much that they can no longer make the $\Delta V \sim 10^{12}$ volts on open field lines needed to sustain a PC accelerator. This is long after they would fail to provide the $\Delta V \sim 10^{14}$ volts needed to sustain large current flow through OG accelerators.

On the other hand, observations of $\gamma$-ray pulsars seem to point to OG accelerators as the needed power source for the following reasons.

a) As long as the OG accelerator spans a major fraction of the open field-line bundle, the total $\gamma$-ray luminosity from the accelerator should be near $j_{\mathrm{GJ}} \Delta V \sim$ $j_{\mathrm{GJ}} \times 10^{14}$ volts. This model result is graphed in Figure 1 . Also shown is the $L_{\gamma}$ from observations, assuming that the integrated intensity over a spin-cycle does 
not depend sensitively on the observer's direction. This would be the case for the fan-shaped beam geometry expected for OG accelerator emission because such accelerators would and should have large latitudinal spreads.

In Figure 1, we see that the "observed" $L_{\gamma}$ are about equal to those calculated to sustain the needed pair production in OG accelerators (from $\gamma+\mathrm{X} \rightarrow$ $\left.e^{-}+e^{+}\right)$. It is almost $10^{2}$ times greater than the maximum power expected from PC accelerators. The few candidate pulsars which could produce OG accelerators with $\Delta V \sim 10^{14}$ volts but were, nevertheless, not observed as strong $\gamma$-ray emitters, may still have nearly the predicted $L_{\gamma}$. The high fraction of candidates which were observed with nearly the predicted $L_{\gamma}$ supports the assumption that observed $\gamma$-ray luminosity does not vary greatly with viewing angle. The data in Figure 1 is, therefore, quite compatible with $\mathrm{OG}$ accelerator properties $\left(\Delta V \sim 10^{14}\right.$ volts, fan beams) but is rather difficult to understand in terms of $\mathrm{PC}$ ones.

b) Pulsar spin-down power $(\dot{E})$ decreases with increasing spin-period more rapidly than does $L_{\gamma}$. These two powers cross near $\dot{E} \sim 10^{34} \mathrm{erg} \mathrm{s}^{-1}$, close to the spin-down power of the Geminga $\gamma$-ray pulsar. In this neighborhood, spinning-down pulsars should be extraordinarily efficient $\gamma$-ray emitters, but when $\dot{E}$ falls significantly below $10^{34} \mathrm{erg} \mathrm{s}^{-1}, \Delta V \sim 10^{14}$ volts can no longer be achieved. Then, OG accelerators can not bootstrap their $e^{ \pm}$production, and their $L_{\gamma}$ should drop precipitously. It may therefore be significant that the radio pulsar PSR 1929, whose maximum OG accelerator $\Delta V$ and current flows are not much below those of Geminga, still has an upper bound to its observed $L_{\gamma}$ which is about two orders of magnitude less than Geminga's. If, as suggested by this bound, the death of powerful $\gamma$-ray pulsars does indeed occur at $\dot{E} \sim 10^{34} \mathrm{erg} \mathrm{s}^{-1}$, this would be crucial support for OG accelerators rather than $\mathrm{PC}$ accelerators as their power sources. The death point $\dot{E}$ for the latter-below which $e^{ \pm}$production is no longer self-sustainable and radio emission ceases-is about 4 orders of magnitude smaller. Because this particular argument for $O G$ accelerators as the source of observed $L_{\gamma}$ depends upon the failure to detect it, the single argument of radio pulsar PSR 1929 will remain weak until there is more data about other pulsars with similar $\dot{E}$.

c) Another argument against PC accelerators as the source of $L_{\gamma}$ may be the failure to see any spectral break from some $\gamma$-ray pulsars, even as $\gamma$-ray energies approach $10 \mathrm{GeV}$ (e.g., PSR 1055-52, Thompson et al. 1999). Such high-energy $\gamma$-rays can escape from the region above a $\mathrm{PC}$ accelerator before $\gamma+\mathbf{B} \rightarrow e^{+}+e^{-}+\mathbf{B}$ conversion only if the $\mathbf{B}$ through that region makes an angle of less than $10^{-1}$ rad with the local (radial) gravitational field. Otherwise, gravitational bending of $10 \mathrm{GeV} \gamma$-ray trajectories would cause them to cross local field lines and be converted to pairs. But there is no special reason for such field configurations (e.g., a central dipole) on the surface of a pulsar, especially when that surface is so close to the current flows which are the source of that field.

d) Finally, there is significant support for an OG accelerator location in the coincidence in phase and light-curve shape of the optical, $\mathrm{X}$-ray, and $\gamma$ ray emissions from the Crab pulsar. The strong optical intensity is roughly consistent with synchrotron radiation from the same $e^{-} / e^{+}$which radiate the $1-10^{3} \mathrm{keV} \mathrm{X}$-rays. Moreover, it is hard to see any other (noncoherent) emission 


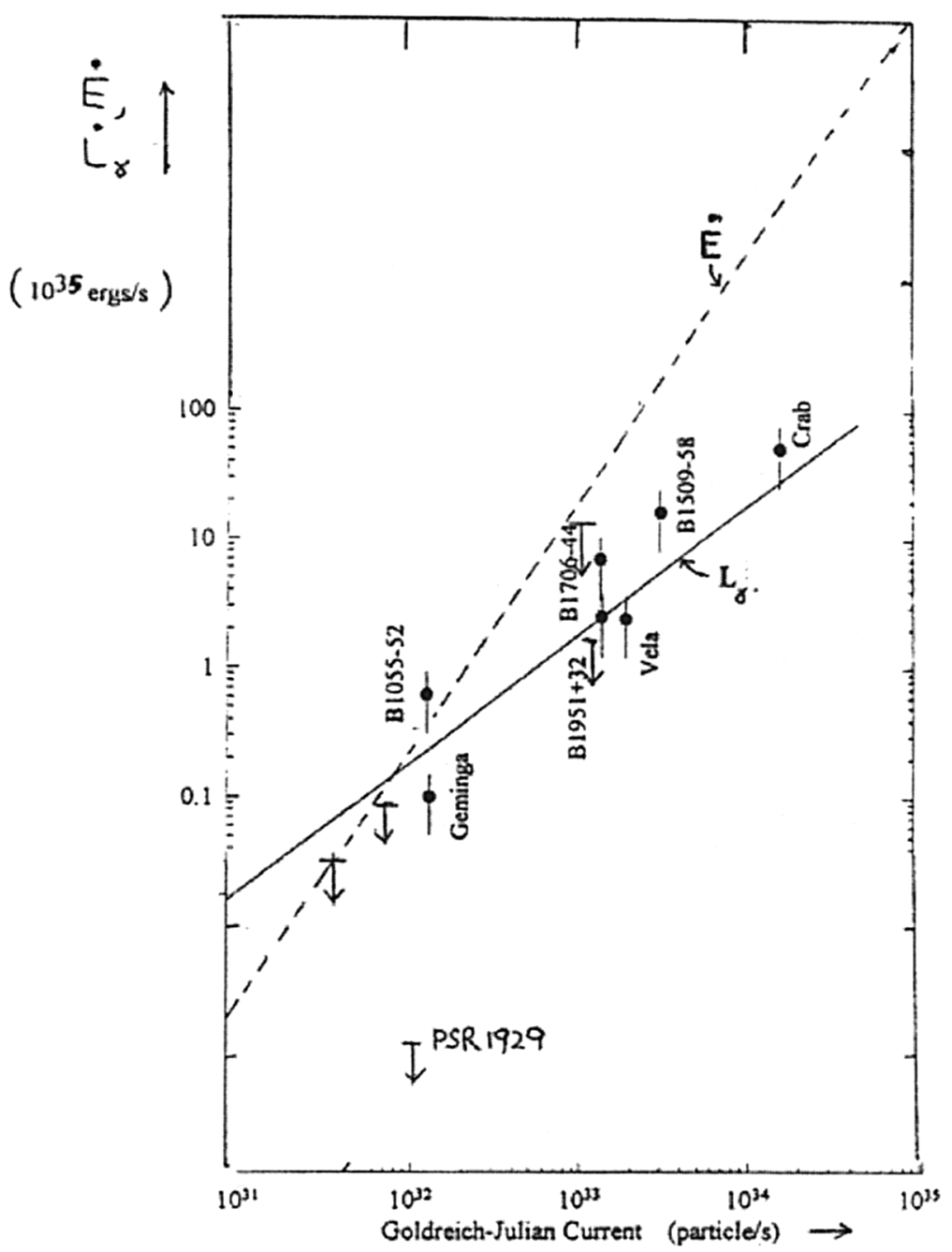

Figure 1. High-energy $\gamma$-ray $(0.1-10 \mathrm{GeV})$ luminosities $\left(L_{\gamma}\right)$ inferred from the observed intensities as discussed in $\S 1$, and total neutron star spin-down power $(\dot{E})$, as a function of total Goldreich-Julian particle current $\left(j_{\mathrm{GJ}} / e\right)$. The solid line is the model's $L_{\gamma}=j_{\mathrm{GJ}} \Delta V$, with $\Delta V=10^{14}$ volts. The dashed line is the total spin-down power $\dot{E}$ calculated for that $j_{\mathrm{GJ}}$. The $\gamma$-ray pulsars designated by label are those detected except for the radio pulsar PSR 1929. Data and figure are based upon Thompson et al. (1999) and references therein. 
process which is efficient enough to give this optical intensity, but this needs a local cyclotron frequency, $e B / m c$, somewhat below the optical region. The required $B$ then puts the radiating $e^{+} / e^{-}$in the outer magnetosphere. The phase coincidence between optical and $\gamma$-ray emission for each of two subpulses then argues strongly for $L_{\gamma}$ also coming from that same region.

In summary, I think that present evidence gives most support for the existence of two distinct accelerator families: 1) an outer magnetosphere (OG) which powers $\gamma$-ray luminosity and is active as long as the total spin-down power $\dot{E} \gtrsim 10^{34} \mathrm{erg} \mathrm{s}^{-1}$; and 2) a polar-cap one which is almost always the generator which powers (coherent) radio emission, as long as $\dot{E} \gtrsim 10^{30} \mathrm{erg} \mathrm{s}^{-1}$.

\section{Accelerator Wars}

These accelerators exist to control charge depletion in some part(s) of a pulsar's open field-line bundle by creating $e^{ \pm}$pairs. The electric fields within them (mainly along B) separate pairs into oppositely flowing, extreme relativistic $e^{-}$ and $e^{+}$. One of these particle beams will emerge from the starward end of an OG accelerator and flow down to the stellar-surface polar-cap area along the open field-line bundle which connects this accelerator to the polar cap. Inside the accelerator, these $e^{-}\left(e^{+}\right)$curvature-radiate $\mathrm{GeV} \gamma$-rays. After they emerge from the accelerator, they continue to emit curvature radiation (about $10^{5} \gamma$ rays for each electron flowing down toward the star), but the characteristic $\gamma$-ray energies will quickly drop to $\sim 10^{2} \mathrm{MeV}$. Those $\gamma$-rays which pass within several neutron-star radii make pairs $\left(\gamma+\mathbf{B} \rightarrow e^{+}+e^{-}+\mathbf{B}\right)$ which would completely quench any polar-cap accelerator onto which they flow.

Alternatively, many of the $e^{ \pm}$pairs made on open field lines by curvature $\gamma$-rays from polar-cap accelerated electrons may flow out along these field lines into the region where an OG accelerator would otherwise have formed. If this is the case, these pairs should quench that accelerator presence since it takes so much weaker an electric field to separate these pairs into oppositely flowing $e^{+}$and $e^{-}$than it does to sustain those processes which create new pairs there. It would be mainly because of gravitational bending of $\gamma$-ray trajectories in a "favorable" direction, or particular magnetic field-line configurations, that PCaccelerator generated pairs could reach OG accelerator locations. In such cases, a PC accelerator should quench any OG accelerator on the same total open field-line bundle. If not, OG accelerators should quench the PC ones.

This mortal struggle for survival among magnetospheric accelerators should result in several possibilities for $\gamma$-ray pulsars. Some will have no radio emission at all and so would be classified today among "unidentified $\gamma$-ray sources" (which outnumber the identified ones). Others could be observable also as radio pulsars only from one side of the star (cf. Figures $2 \mathrm{a}, \mathrm{b}$ ). From the other side, although that is where the OG accelerator is, the observability of $\gamma$-rays depends upon details of the number and geometry of $\gamma$-ray beams from an OG accelerator. In the OG models presented at this meeting by Romani (2000) and by Cheng, Ruderman, \& Zhang (2000), $\gamma$-ray beams would not be observed (Figure 2b), but, in that of Hirotani (2000), they probably would (Figure 2a). 


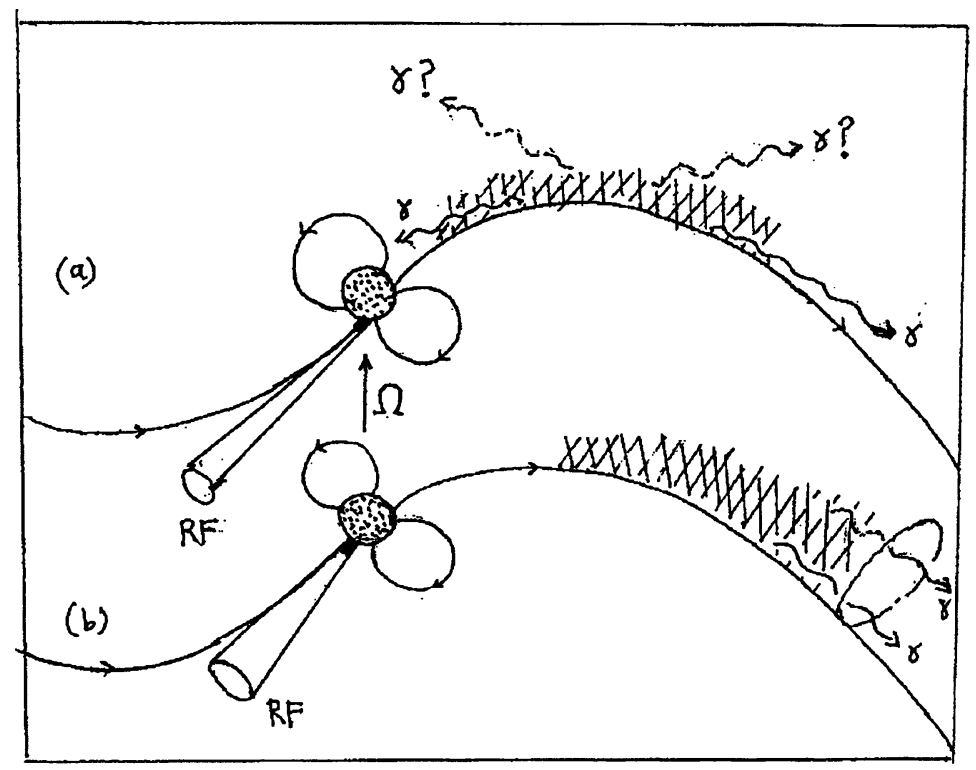

Figure 2. Cartoon of possible OG (cross-hatch) and PC (solid fill) accelerator positions in an $\gamma$-ray pulsar. It is assumed that, as discussed in $\S 2$, only one survives on each side of the star. Different conceivable possibilities for $\gamma$-ray beam geometries are indicated in a) and b).

\section{Speculations About Transient Accelerators}

In addition to the rich and unique landscape of energetic particle accelerators within corotating pulsar magnetospheres, special properties of neutron-star interiors might sometimes result in remarkably enormous, transient fluxes of energetic radiation. I will present one such possibility.

White dwarfs (WDs) accreting from a surrounding accretion disk fed by a companion grow to exceed the maximum possible WD mass about once every 500 years in our Galaxy. It then begins to collapse and become hotter. If the WD is not very highly evolved, e.g., with a $\mathrm{C}, \mathrm{N}$, and $\mathrm{O}$ composition, this results in an enormous nuclear-fusion-powered explosion. This is the familiar, observed Type I supernova, which leaves no remnant; but, if the accretioninduced collapse is that of a more highly evolved WD consisting of $\mathrm{O}, \mathrm{Ne}$, and $\mathrm{Mg}$, electron capture during the initial collapse removes enough pressure support that a neutron star remnant is expected (Nomoto \& Kondo 1991). The initial spin of this remnant neutron star depends upon how much angular momentum was transferred to the WD as a result of its accretion. This spin-rate can be close to the maximum $10^{3} \mathrm{~Hz}$ of a neutron star for plausible WD accretion rates and durations. Such a newly born (hot) neutron star would also begin its life with a huge amount of kinetic energy (up to $10^{53} \mathrm{ergs}$ ) in differential rotation. This is 
a consequence of the equation of state of its parent WD just before its collapse. The WD's pressure support comes from degenerate relativistic electrons which give a pressure proportional to (density) ${ }^{4 / 3}$. Because of this, the spinning, preimplosion WD has a central density 50 times its average density. This ratio is over an order of magnitude larger than the ratio of central-to-average-density in the neutron star which it becomes. The accretion-induced collapse is extremely nonhomologous: the initially hot neutron star begins its life with its outer parts spinning much more rapidly than its core. In the absence of any strong, internal magnetic field, most of this differential rotation could survive the initial rapid neutrino cooling of the neutron star and easily remain for hours or longer, but $\sim 10^{-1}$ of WDs are strongly magnetized with $B \gtrsim 10^{9} \mathrm{G}$. Accretion-induced collapse would then be expected to give birth to a magnetized neutron star with $B \geq 10^{12} \mathrm{G}$. This $B$ would couple differentially rotating cylinders (radius $\left.r_{\perp}\right)$ in the neutron star, spinning with different angular speeds $\left(\Omega\left(r_{\perp}\right)\right)$ about a common spin axis. The magnetic field which connects them would then begin to wind-up into a growing toroidal $B_{\mathrm{T}}$. Magneto-rotational instabilities which could cause exponentially fast dissipation of the differential rotation energy into turbulent magnetic field do not preempt the steady toroidal wind-up since

$$
\frac{\mathrm{d}}{\mathrm{d} r_{\perp}}\left|\Omega\left(r_{\perp}\right)\right|>0
$$

(Balbus \& Hawley 1998); but, as $B_{\mathrm{T}}$ grows, so does magnetic buoyancy. In a cooled neutron star (but not yet a superfluid one), this buoyancy would be strongly opposed by the almost frozen stratification of the $(e+p)$-to- $n$ ratio inside the star. This antibuoyancy would not be effective in the first 10 or so seconds after the birth of this neutron star if it is born with a temperature of several $\mathrm{MeV}$ or more: until its neutrino temperature drops, $n /(e+p)$ is too quickly adjusted by $e+p \leftrightarrow n+\nu$ (Thompson 2000). However, after about 10 seconds, almost all of these neutrinos would have escaped, and stratification (and also, perhaps, thermal gradients) must be overcome before buoyant, wound-up magnetic toroids would finally rise up to and burst through the stellar surface. It has been estimated by Kluźniak \& Ruderman (1998) that the toroidal $B_{\mathrm{T}}$ will grow to exceed $10^{17} \mathrm{G}$ before this happens and the magnetic energy in the torus reaches $\sim 10^{52}$ ergs. Wind-up and expulsion through the stellar surface could repeat at characteristic intervals of 10 seconds (for an initial $B \sim 10^{12} \mathrm{G}$ ) until there is no longer enough energy left in the remaining differential rotation for yet another complete wind-up and expulsion. It was, of course, noted that the time scales between sub-bursts, total durations, and energy releases are suggestive of those needed for the central engines of cosmic Gamma-Ray Burst sources.

This particular speculation is an example of the huge, transient power for ultimate particle acceleration which might come from a neutron star if we consider rare events (e.g., one per $10^{6}$ years per Galaxy, see Usov 1992). Until the origin(s) of cosmic GRBs is understood, there will almost certainly be proposed models for them in which neutron stars are an essential part. 


\section{Concluding Remarks}

The origin of the magnetospheric accelerators of Sections 1 and 2 is thought to be charge depletion in some part of the open field-line bundle in a newly corotating neutron-star magnetosphere; accelerator modelers have generally assumed $\partial \mathbf{B} / \partial t=0$ (perhaps too cavalierly). It is a mechanism for particle accelerators which may not have close analogues among the other astronomical systems considered at this conference. In the neutron-star model for the conversion of stellar implosion and rotational energy into an enormous, transient power source for particle acceleration, it is crucial that the underlying star is supported by the pressure of very stiff matter. For all of these neutron-star accelerators, there are not expected analogues among accretion disks, black holes, Active Galactic Nuclei, or the surface of the sun and that of other conventional stars. This is unfortunate because recent growing insights about these have not much informed the understanding of acceleration phenomena in isolated neutron stars.

The happy part is that what has been an engaging enterprise for three decades does not seem about to end.

\section{References}

Balbus, S., \& Hawley, J. 1998, Rev. Mod. Phys., 70, 1

Cheng, K. S., Ruderman, M., \& Zhang, L. 2000, these Proceedings

Hirotani, K. 2000, these Proceedings

Kluźniak, W., \& Ruderman, M. 1998, ApJ, 505, L113

Lyutikov, M., Blandford, R., \& Machabeli, G. 2000, these Proceedings

Nomoto, K., \& Kondo, Y. 1991, ApJ, 367, L19

Pacini, F. 2000, these Proceedings

Romani, R. W. 2000, these Proceedings

Thompson, C. 2000, these Proceedings

Thompson, D., et al. 1999, ApJ, 516, 297

Usov, V. 1992, Nature, 357, 472 